\title{
The application and improvement of Fourier transform spectrometer experiment
}

Zhi-min Liu, En-duo Gao, Feng-qi Zhou, Lan-lan Wang, Xiao-hua Feng, et al.

Zhi-min Liu, En-duo Gao, Feng-qi Zhou, Lan-lan Wang, Xiao-hua Feng, Jinquan Qi, Cheng Ji, Luning Wang, "The application and improvement of Fourier transform spectrometer experiment," Proc. SPIE 10452, 14th Conference on Education and Training in Optics and Photonics: ETOP 2017, 104524N (16 August 2017); doi: 10.1117/12.2268874

SPIE Event: 14th Conference on Education and Training in Optics and Photonics, ETOP 2017, 2017, Hangzhou, China 


\title{
The Application and Improvement of Fourier transform Spectrometer Experiment
}

\author{
Liu Zhi-min*, Gao En-duo, Zhou Feng-qi*, Wang Lan-lan, Feng Xiao-hua, Qi \\ Jin-quan, Ji Cheng, Wang Luning \\ Department of Optoelectronic Information Science and Engineering, School of \\ Science, East China Jiaotong University, Nan chang 330013 China \\ Liuzhimin2006@163.com,Zhoufengqi2004@163.com
}

\begin{abstract}
According to teaching and experimental requirements of Optoelectronic information science and Engineering, in order to consolidate theoretical knowledge and improve the students practical ability, the Fourier transform spectrometer ( FTS) experiment, its design, application and improvement are discussed in this paper. The measurement principle and instrument structure of Fourier transform spectrometer are introduced, and the spectrums of several common Laser devices are measured. Based on the analysis of spectrum and test, several possible improvement methods are proposed. It also helps students to understand the application of Fourier transform in physics.
\end{abstract}

Keywords: Fourier transform; Spectrum; Experiment.

\section{INTRODUCTION}

Fourier Transform Spectrometer (FTS) ${ }^{[1]}$ is based on the Fourier transform of the measured interference pattern to obtain spectrum. It has many advantages, such as multi-channel, high throughput, high precision, high signal to noise ratio, wide spectrum, non-contact, digital and so on ${ }^{[2,3]}$. FTS is very important for the study of atomic and molecular physics, astronomy and physics, spectroscopy, atmospheric remote sensing, analytical chemistry and other fields ${ }^{[4-6]}$. And it is also essential equipment for industrial inspection, customs inspection and so on ${ }^{[7]}$.

Michelson interferometer and Fourier Transform Spectrometer are the instruments of measuring wavelength which is used frequently in college physics experiment teaching. The studies of Fourier Transform Spectrometer focus on the aspects of optical path difference ${ }^{[8]}$, resolution ${ }^{[9]}$, dynamic correction technique ${ }^{[10,11]}$, Static Fourier Transform Spectrometer ${ }^{[12,13]}$. There have been many patents in domestic ${ }^{[14]}$.

In this paper, the measurement principle and instrument structure of Fourier transform spectrometer are introduced, and the spectrums of several common Laser devices are measured. Based on the analysis of spectrum and test, several possible improvement programs are proposed. It helps students to understand the application of Fourier transform in physics.

14th Conference on Education and Training in Optics and Photonics: ETOP 2017, edited by Xu Liu,

Xi-Cheng Zhang, Proc. of SPIE Vol. 10452, 104524N · C 2017 ICO, IEEE, OSA, SPIE

CCC code: $0277-786 X / 17 / \$ 18 \cdot$ doi: $10.1117 / 12.2268874$

Proc. of SPIE Vol. $10452104524 \mathrm{~N}-1$ 


\section{THE EXPERIMENTAL PRINCIPLE OF FTS ${ }^{[15,16]}$}

Fourier transform spectroscopy is used to modulate the incident light by Michelson interferometer. An interferometric signal is changed into an electrical signal by a detector converts, and digital time domain interferogram is obtained by A/D. There is a Fourier transform relation between time domain interferogram and the incident light spectrum. The spectral information of incident light can be obtained by Fourier inverse transform of interferogram.

Fourier transform are the process of modulation and demodulation in fact, modulation is realized by Michelson interferometer, and the intensity value of the interference is

$$
\mathrm{I}(\mathrm{x})=\int_{-\infty}^{\infty} I(\sigma) \cos 2 \pi \sigma x d \sigma
$$

Where: $\mathrm{x}$ is the optical path difference; it moves with the moving mirror, $\sigma$ is the wave number for monochromatic light.

Since the Fourier transform is reversible, if measured light is continuous spectrum, the spectral intensity distribution after interference can be calculated as following:

$$
\mathrm{I}(\sigma)=\int_{-\infty}^{\infty} I(\mathrm{x}) \cos 2 \pi \sigma x d \mathrm{x}
$$

The optical path difference of the interferometer is changed continuously, and the variable optical flux emitted by an interferometer can be recorded by an optoelectronic element. So the radiation intensity of any wave number can be obtained by Fourier cosine transform of Interferogram.

\section{EXPERIMENT AND RESULTS}

\subsection{Experimental device}

XGF-1 type Fourier transform spectrometer is used in this paper, the integrated device is shown in Figure1, light path and acquisition system, computer software system, an external light source are included.

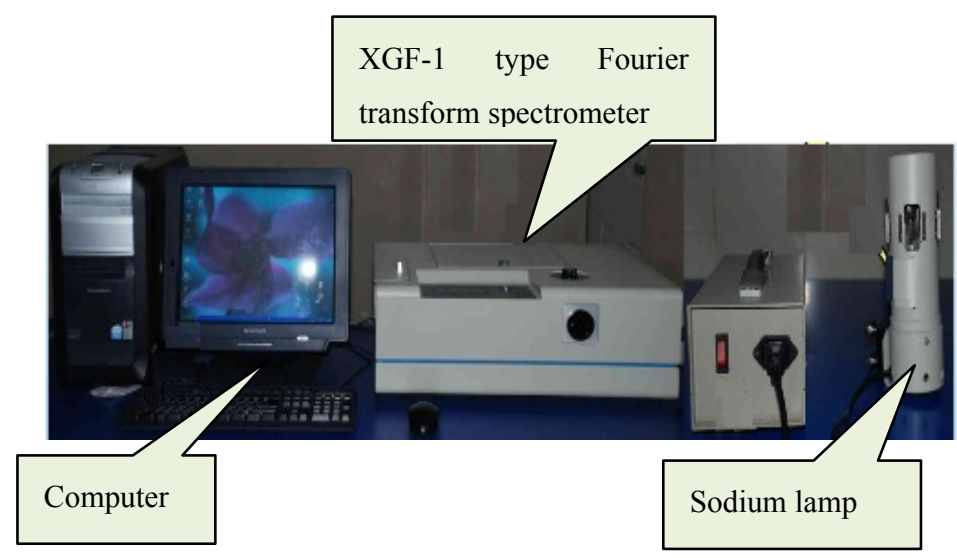

Figure 1 XGF-1 type Fourier transform spectrometer 


\subsection{Optical path}

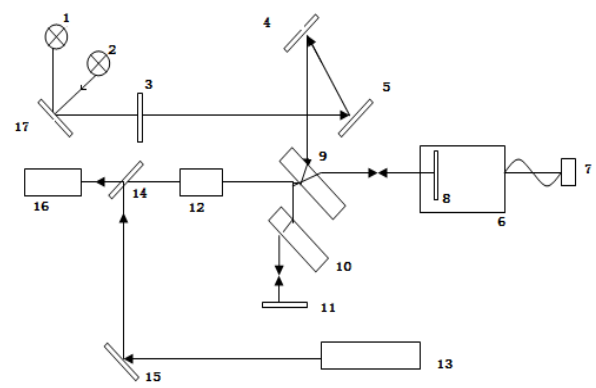

Figure 2 Internal optical path of FTS (1-external source, 2-built-in light source, 3- adjustable diaphragm, 4- collimation mirror, 5- Plane mirror, 6- precision translation stage, 7- slow motor, 8- movable mirror,

9- Interference plate 10- compensation plate, 11- Fixed mirror, 12- receiver 1 13-Reference light source 14-, t Semitransparent mirror 15- plane mirror, the 16- receiver 2, 17- light source converter)

The XGF-1 type FTS is a time modulation type moving mirror spectrometer, which is obtained by Fourier transform of the interference information generated by the double beam interferometer. The core part is the Michelson interferometric optical system, light path is shown in figure 2. The parallel light passing through the collimating mirror enters semitransparent mirror of the interferometer and divides into two coherent lights with certain phase difference. When the moving mirror of the interferometer moves continuously, the changes of interferogram will be received by receiver and recorded at a certain data intervals by the recorded system.

\subsection{Results}

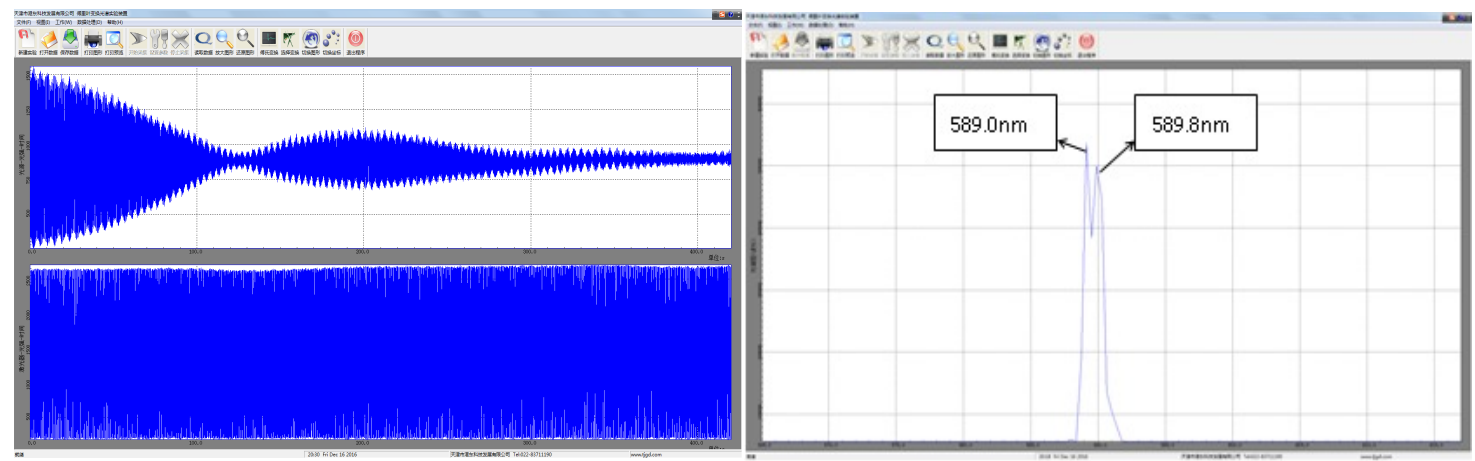

Figure 3 The interferogram and Spectrum of Na lamp

Firstly we preheat the experimental instrument and do other preparations, the light column diameter and acquisition time are set to $2.6 \mathrm{~mm}$ and 7 minutes, and the magnification of the light source is set to "*16" file. The collected interferogram shows the shape of the gourd, as shown in Figure 3(left). The reasons are as follows:

The actual light sources in interferometry are not absolute monochromatic but have a certain spectral line width $\Delta \lambda$. Therefore, the fringe intensity of other wavelengths are distributed between the two curves $\lambda$ and $\lambda+\Delta \lambda$. The actual fringes in the interference field are the superposition of these interference fringes. The maximum value of each wavelengths coincides in the zero level, then 
slowly staggered, staggered distance of the maximum value of each wavelengths increases when the interference level is higher, the bonding strength of the peak and the contrast ratio decrease gradually. We take a rectangular spectral structure with bandwidth as an example. The intensity distribution of all the lines in the interference field is:

$$
\mathrm{I}=\int_{\mathrm{k}_{0}-\frac{\Delta \mathrm{k}}{2}}^{\mathrm{k}_{0}+\frac{\Delta \mathrm{k}}{2}} 2 \mathrm{I}_{0}(1+\cos k \Delta) d k=2 I_{0} \Delta k\left(1+\frac{\sin \left(\Delta k \cdot \frac{\Delta}{2}\right)}{\Delta k \cdot \frac{\Delta}{2}} \cos \left(k_{0} \Delta\right)\right)
$$

Where $\mathrm{k}_{0}=\frac{2 \pi}{\lambda}, \Delta_{\max }=\frac{2 \pi}{\Delta k}=\frac{\lambda_{1} \lambda_{2}}{\Delta \lambda} \approx \frac{\lambda^{2}}{\Delta \lambda}$.

The reason of the gourd shape in the interferogram is the decrease of interference contrast ratio.

The interferogram is transformed by Fourier transform of rectangular function and spectrum is local amplified, which is shown in Figure 5(right), we can directly get the wavelengths of Na lamp, one is $589.0 \mathrm{~nm}$, and the other is $589.8 \mathrm{~nm}$. The relative errors between the standard values $589.0 \mathrm{~nm}$ and $589.6 \mathrm{~nm}$ are as follows respectively:

$$
\begin{gathered}
\eta_{1}=\left|\frac{589.0-589.0}{589.0}\right| \times 100 \%=0 \% \\
\eta_{2}=\left|\frac{589.8-589.6}{589.6}\right| \times 100 \%=0.034 \%
\end{gathered}
$$

The results indicate that the wavelength of the Na light can be measured accurately; the experimental results meet the requirements of resolution.

Similarly, the light column diameter and acquisition time are set to $6.0 \mathrm{~mm}$ and 7 minutes, and the magnification of the light source is set to "*16" file. Double yellow lines of the measured Na lamp are $589.4 \mathrm{~nm}$ and $590.2 \mathrm{~nm}$, the relative errors are $0.068 \%$ and $0.102 \%$ respectively. In comparison, the diameter of the light bar has a great influence on the results.

\subsection{Applications}

We use this instrument to measure the spectrum of several common light sources in the laboratory.
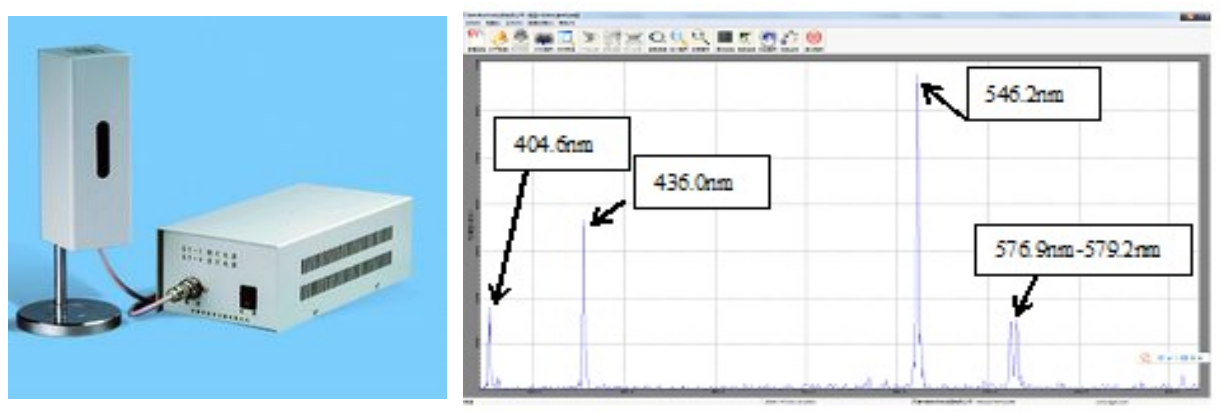

Figure 4 light source of high pressure mercury lamp and the spectrum

High pressure mercury lamp light source and the spectrum are shown in Figure 4. The main 
radiations are $404.7 \mathrm{~nm}, 435.8 \mathrm{~nm}, 546.1 \mathrm{~nm}$ and $577.0 \sim 579.0 \mathrm{~nm}$ in visible spectral line. Experimental results show the wavelengths of experimental results are $404.6 \mathrm{~nm}, 436.0 \mathrm{~nm}, 546.2 \mathrm{~nm}$ and $576.9 \sim$ $579.2 \mathrm{~nm}$, the spectrums of the visible part are measured accurately.

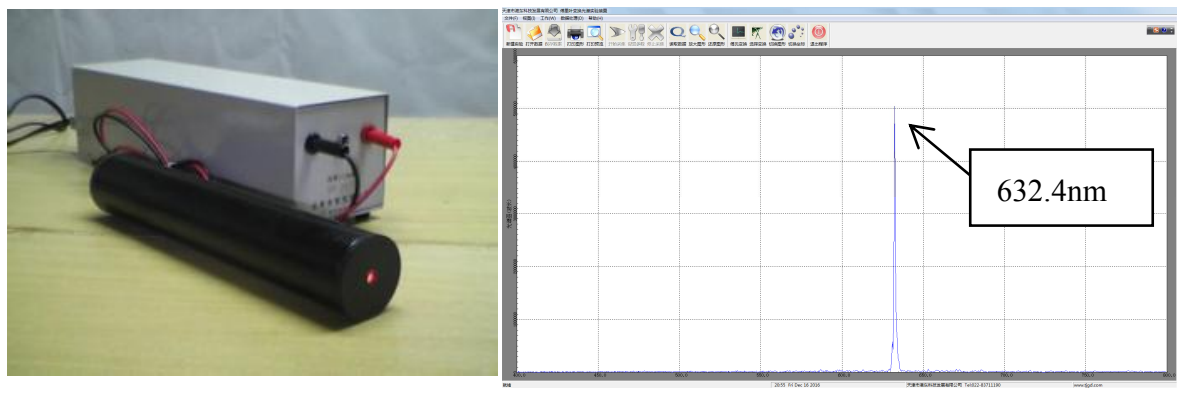

Figure 5 Light source and spectrum of He-Ne laser

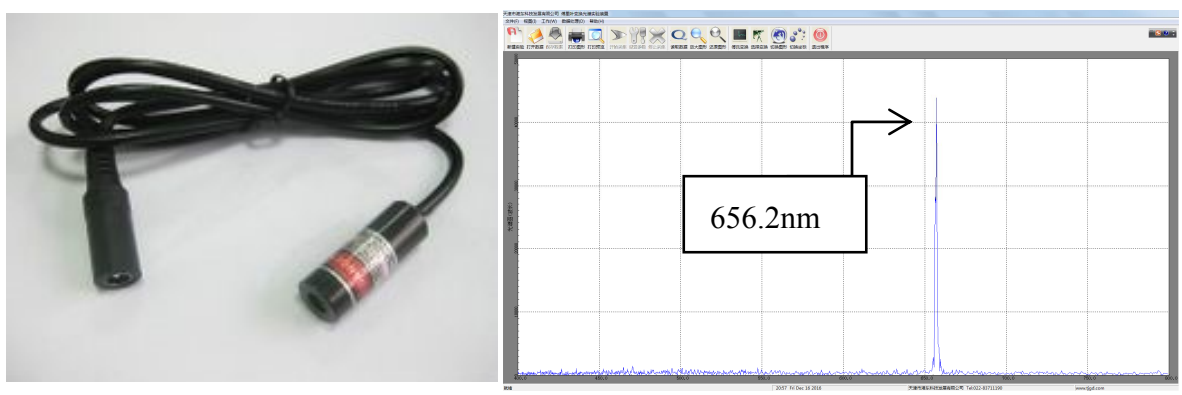

Figure 6 Light source and spectrum of red semiconductor laser

The spectrum of He-Ne laser and red semiconductor laser are shown in Figure 5 and 6 . The wavelengths of experimental results are $632.4 \mathrm{~nm}$ (theoretical value of $\mathrm{He}-\mathrm{Ne}$ laser is $632.8 \mathrm{~nm}$ ) and $656.2 \mathrm{~nm}$ (theoretical value of red semiconductor laser is $650.0 \mathrm{~nm}$ ), Experimental results show the spectrums of the visible part are measured accurately.

\section{EXPERIMENTAL IMPROVEMENTS}

\subsection{Methods for improving resolution}

From the expression of resolution ${ }^{[17]}$ :

$$
R=\frac{\lambda}{\delta \lambda_{m}}=\frac{21_{0}}{\lambda}
$$

We know that the resolution of spectrum is proportional to the maximum moving distance of the moving mirror. In order to obtain high resolution, the moving mirror is required to move a larger distance, and the larger the moving distance is, the harder the interferometer is made. Obviously, the Michelson interferometer cannot meet the requirement of resolution completely. Therefore, it is necessary to change the design of the spectrometer, larger optical path difference can be obtained just by moving a short distance of the moving mirror. An interferometer can better meet this requirement as following description: 
The improved structure is shown in Figure 7. The conventional moving mirror is changed to a group of movable mirrors consisted by a right angle moving mirror and a plane mirror (Refraction-Refraction mirror), the Refraction-Refraction mirror is fixed, and right angle mirror can be moved. The light from the light source passes through the beam splitter, one of the lights is shot on the Right angle moving mirror firstly, and then reflected back from the Refraction-Refraction mirror. The optical path of the light increases rapidly due to the existence the group of mirrors, the group of mirrors only moves a small distance, we can get a large optical path difference. The design of this kind of structures can improve the resolution.

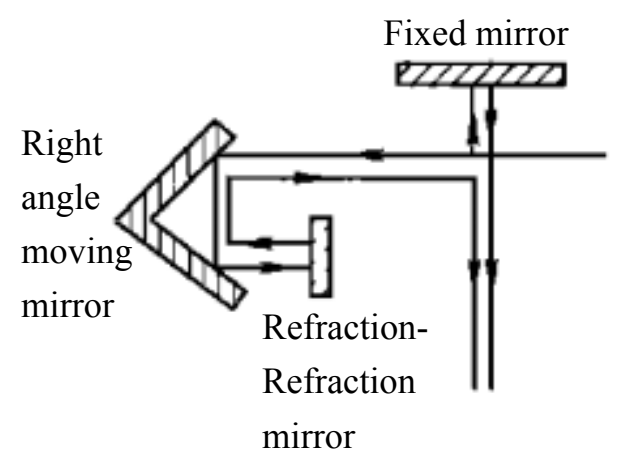

Figure7 Refraction-Refraction type interferometer

\subsection{Improvements of the experimental instrument structure}

Firstly, the XGF-1 type Fourier transform spectrometer is only equipped with eight fixed apertures, as shown in Figure 8. Light bar diameter changes from $1.4 \mathrm{~mm}$ to $6.0 \mathrm{~mm}$ increasing by $0.4 \mathrm{~mm}$ increments. But according to the formula $R=\lambda / \delta \lambda_{m}=21_{0} / \lambda$, the resolution is closely related to the actual wavelength, and also according to the results of above measurements, the diameter of the apertures has a great influence on the accuracy of the results. Therefore, we think that the apertures can be adjusted to continuously adjustable circular hole, as shown in Figure 9.

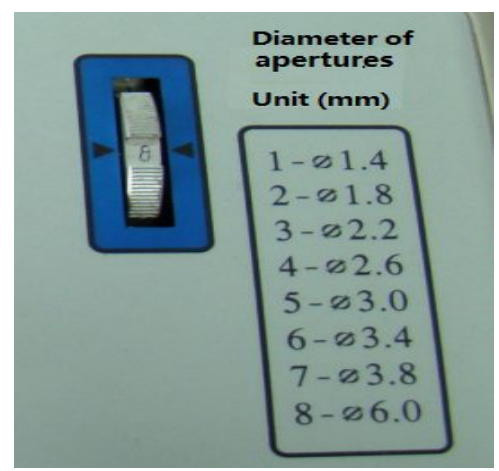

Figure 8 Light aperture switch

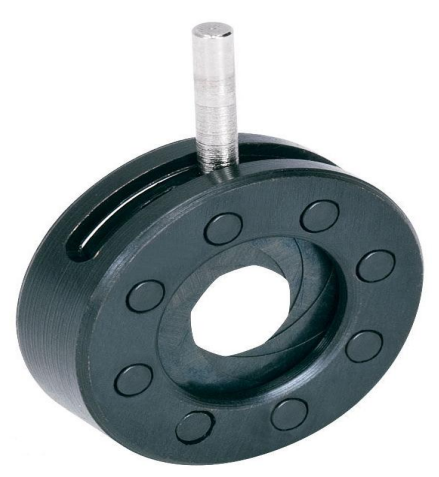

Figure 9 Improved light aperture

The light source switch of XGF-1 type Fourier transform spectrometer is shown in Figure 10, light source switch only has two choices: bromine tungsten lamp and other light sources. However, the directivity of the incident light directly affects the alignment of the optical path, and the light passing a distance will produce some errors, thus the difficulty of the experiment will be increased. The 
following improvements are made for the light source switch: The light source converter can be designed as a $360^{\circ}$ continuous adjustable and rotatable reflector, which is easy to fix at a single column support, as shown in Figure 11. Thus, when the external light source is fixed or is inconvenient to move, the optical path can be adjusted by rotating the knob of light source switch, so as to the collimation of the light path can be realized better.

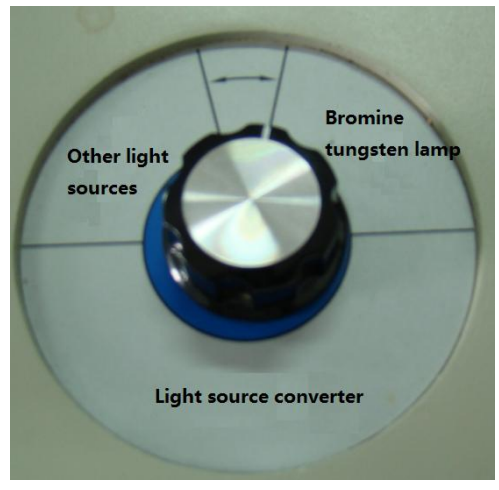

Figure 10 Light source switch

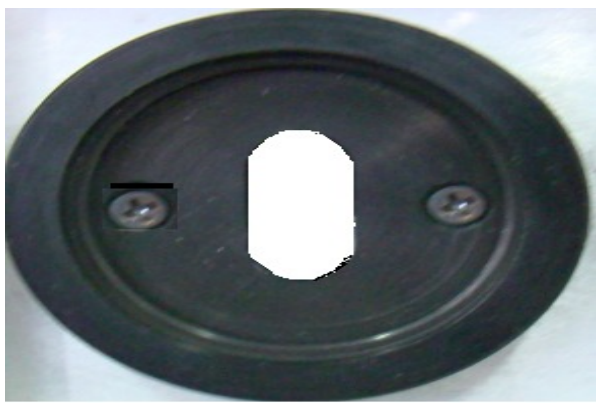

Figure 12 The aperture of external light source

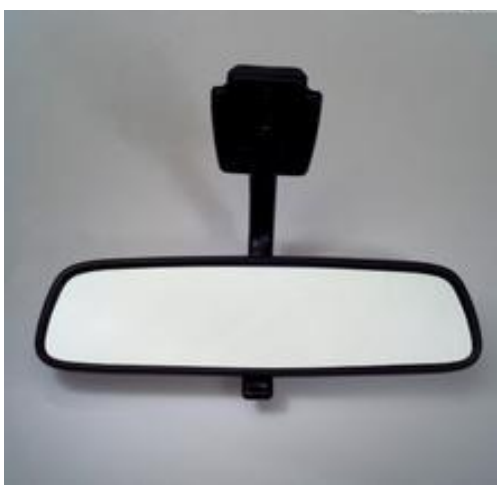

Figure 11 Improved light source mirror

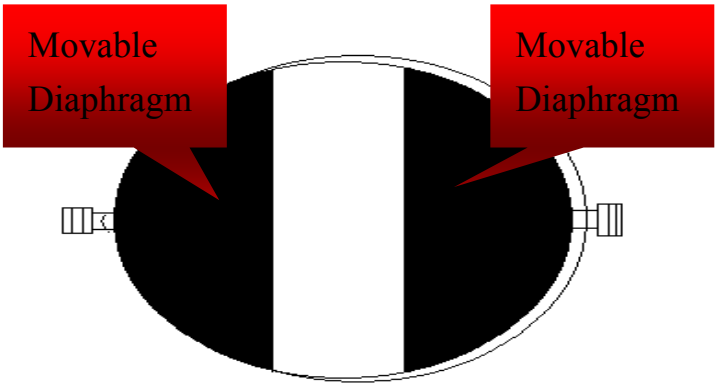

Figure 13 Improved graph of Light aperture

The aperture of external light source is designed to be a rounded and rectangle aperture, shown in Figure 12. The size of aperture directly affects the size of the incident light flux and the quality of the light interference. So we do the following adjustment to the light source aperture: two movable diaphragms are designed, and then the incident light flux can be adjusted continuously. The improved graph of light aperture is shown in Figure 13.

\section{CONCLUSION}

In summary, we use XGF-1 type Fourier transform spectrometer to measure some spectrums of several common light-emitting devices. And multiple reflection interferometers by changing the design of the optical path are proposed to obtain greater optical path difference and improve the resolution of the instrument. At the same time, some improvements were also proposed in the design of the spectrometer to ensure that external factors impact the experimental results minimally. It can help students to understand the application of Fourier transform in physics better. We expect that our study and discussion will lead to significant advances and new opportunities in the design and optimization of Fourier transform spectrometer. 


\section{ACKNOWLEDGMENTS}

This work was funded by the National Natural Science Foundation of China (No. 11164007), the Scientific Project of Jiangxi Education Department of China (No. GJJ160532).

\section{REFERENCES:}

[1] Bell R J, "Introductory Fourier transform spectroscopy," Academic Press, Beijing, 18(1972).

[2] Libo Zeng, Bansheng Yin, Bin He, "Error analysis of moving mirror in Fourier transform interferomete r," Optics and precision engineering, 14 (2) , 191-196 (2006).

[3] Suning Li, Rihong Zhu, Jianxin Li, "Method of reconstruction on Fourier Transform spectroscopy," Journal of Applied Optics,30(2), 268-272(2009).

[4] Bin Yang, "Determination of Emission Concentration of Catering Oil Smoke by FT -IR Spectrometry," Chinese Journal of Spectroscopy Laboratory, 25 (4), 578-580(2008).

[5] Nan Cao, "Analysis of Structure Characteristics of Oxide by Infrared Spectrometer," Chinese Journal of Spectroscopy Laboratory, 24 (6) , 1099-1101(2007).

[6] Lyndin N M, Parriaux O, Tishchenko A V, "Modal analysis and suppression of the fourier modal method instabilities in highly conductive gratings," Journal of the Optical Society of America A, 24 (12), 3781-3788(2007).

[7] Lin Lin, Zhao Ren,Gang Li, "Theory and Correction of Interference Fringe in Static Fourier Transform Spectrometer," Spectroscopy and spectral analysis,28(9), 2067-2072(2008).

[8] Dongqing Zhang, Fuquan Wu, Shuhai Fang, "Analysis of Path Difference about a Fourier Transform Spectrometer Based on Savert Prisms," Journal of Optoelectronics· Laser ,14 (4), 432-434(2003).

[9] XinRong Chen, "A new explanation to the theory resolution power of Fourier transform spectrometer," Journal of Suzhou University ,16 (2), 45-48(2000).

[10] Bansheng Yin, Libo Zeng, Junfeng Lei, "Error analysis of the mirror tilting in spectrum transform interferometer and the study on dynamic alignment technology," Optical instruments,28(1), 74-79(2006).

[11] Fang Sun, Zuoxiao Dai, Jianwen Hua, "A Speed Measuring System of Moving Mirror for spectrum transform interferometer," Semiconductor optoelectronic, 28(2), 283-286(2007).

[12] Jiang Chengzhi, Liang Jingqiu, Liang Zhongzhu, "Influence of collimation system on static fourier transform spectrometer," Spectroscopy and spectral analysis,34(1), 274-278(2014).

[13] Jinguang Lu, Jingqiu Liang, Zhongzhu Liang, "Study on Interferogram-Spectrum Modulation by the Rotation of Compensation Plate in Static Fourier Transform Spectrometer," Acta Optica Sinica, 35(11), 1130001:1-8(2015).

[14] Kejia Wei, "Patent introduction of Fourier transform spectroscopy in domestic," Journal of Science and Technology Economic, 7, 207-209(2016).

[15] Yongchun Yin, "Modern interferometric technique," Tianjin University press, 425-426 (1999).

[16] Guoan Wu, "Spectral instrument design," Beijing: Science and Technology Press,467-470(1978). 
[17] Binai Zuo, Wenbo Li,Yuexiang Peng, "Analysis of Resolution on Fourier Transform Spectrum," Journal of Northern Jiaotong University,27(3), 67-69(2003).

Proc. of SPIE Vol. 10452 104524N-9

Downloaded From: https://www.spiedigitallibrary.org/conference-proceedings-of-spie on 26 Apr 2023 Terms of Use: https://www.spiedigitallibrary.org/terms-of-use 